\title{
The probability distribution of the conductance at the mobility edge
}

\author{
Marc Rühländer ${ }^{1}$ and C. M. Soukoulis ${ }^{1,2}$ \\ ${ }^{1}$ Ames Laboratory and Department of Physics and Astronomy \\ Iowa State University, Ames, Iowa 50012 \\ ${ }^{2}$ Research Center of Crete and Department of Physics \\ University of Crete, Heraklion, Crete, Greece
}

\begin{abstract}
The probability distribution of the conductance $p(g)$ of disordered $2 \mathrm{~d}$ and $3 \mathrm{~d}$ systems is calculated by transfer matrix techniques. As expected, $p(g)$ is Gaussian for extended states while for localized states it is $\log$-normal. We find that at the mobility edge $p(g)$ is highly asymmetric and universal.
\end{abstract}

In the presence of disordert, a system may undergo a transition from insulating to metallic behaviour as the Fermi energy varies in an energy range containing both localized and extended states, seperated by a mobility edge. This transition can be characterized either by transport properties like e.g. the conductance, or by properties of the eigenstates of the system like e.g. the correlation length $\xi_{c}$ (approaching from the metallic side of the transition) or the localization length $\xi_{l}$ (approaching from the insulating side of the transition). While the latter are self-averaging quantities, i.e. the ensemble average may be used as a scaling variable, the conductance is not 2 . Therefore it is of great importance to determine the complete probability distribution $p(g)$ of the conductance $g$ (in units of $e^{2} / h$ ), especially at the critical point of the metal-insulator transition, as it is well known to be a Gaussian on the metallic side and $\log$-normal on the insulating side. The correct form of $p_{c}(g)$ the distribution of the conductance at the mobility edgel 3 , is still not sufficiently well-known.

Using a tight-binding model 3 with diagonal disorder, a transition from a metallic state to an insulating one can be inducede in a finite size sample by increasing the disorder strength $W$. In all our results, $W$ is given in units of the hopping integral. The localization length $\xi_{l}$ decreases as the strength of the disorder, $W$, increases. As long as $\xi_{l}$ is much bigger than the system size, the electron will cross the sample with ease, thus being essentially delocalized. If, on the other hand, $\xi_{l}$ is sufficiently smaller than the system size, the electron will become localized in a small region and not contribute much to the conductance. The critical strength of disorder $W_{c}$ will occur where the localization length becomes comparable to the system size. We have systematically studied the conductance $g$ of the $2 \mathrm{~d}$ and $3 \mathrm{~d}$ tight-binding model by using the transfer matrix technique, which relates $g$ with the transmission matrix $\mathbf{t}$ by $g=2 \operatorname{Tr}\left(\mathbf{t}^{\dagger} \mathbf{t}\right)$. The $g$ defined here is for both spin orientations. Fig. 2 shows the distributions of the conductance $g$ and the natural logarithm of the conductance $\ln (g)$ for a metallic and a localized $2 \mathrm{~d}$ sample respectively. In the "metallic" regime (it is really weakly localized, since we are working in $2 \mathrm{~d}$ ) where $W=3.0$ we have $\xi_{l}=47234$, which is much larger than the system size $L=64$. In the localized regime, where $W=9.0$, we have $\xi_{l}=7.54$, which is smaller than the size of the system. Both distributions fan be fitted very well by a Gaussian normal distributiont. Fig. 1 displays the distributions for a system near the critical point. The distribution of $\ln (g)$ shows a characteristic cut-off at $\ln (g)=0$. Only a few samples have a conductance $g>1$. This is in agreement with analytical results 5 .

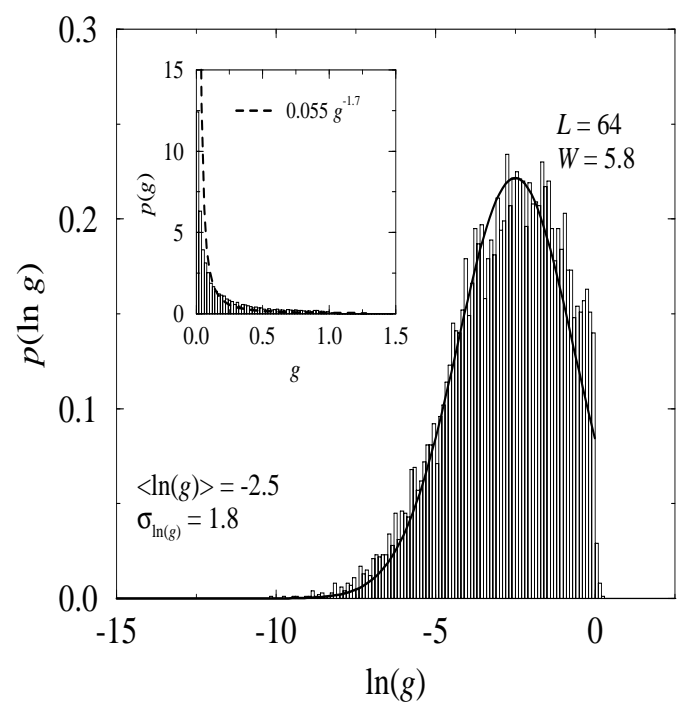

FIG. 1. The distribution of the conductance for a square system of 64 by 64 lattice sites. The disorder was chosen such that the localization length is close to the system size $L$. 

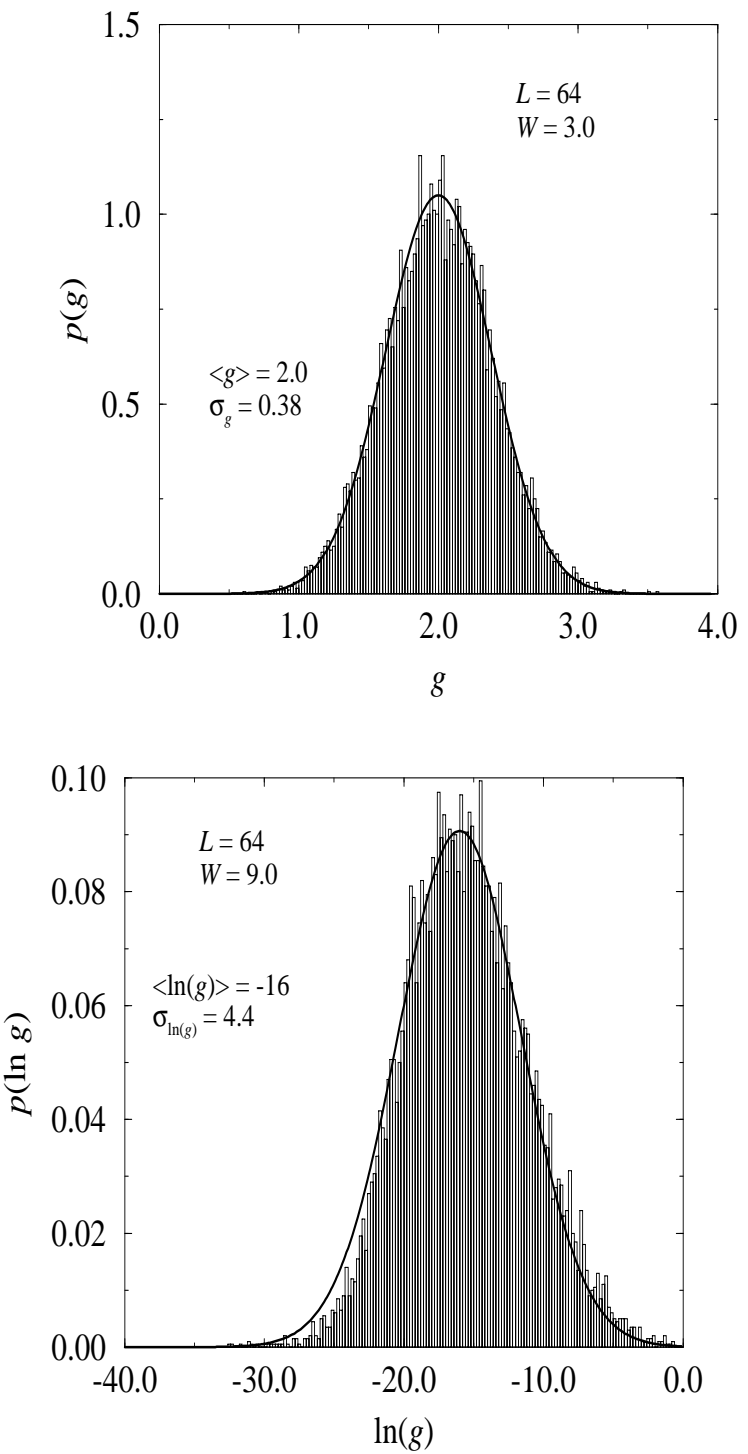

FIG. 2. The distribution of the conductance for a square of 64 by 64 lattice sites. Small disorder ( $W=3$, top panel) leads to metallic behaviour; strong disorder ( $W=9$, bottom panel) leads to insulating behaviour. The values for the average and standard deviation of the Gaussian fits are given in the graphs.

Applying a strong magnetic field perpendicular to a $2 \mathrm{~d}$ system creates states with a diverging localization length $\mathrm{\theta}$ even in the thermodynamical limit, as long as the disorder is not too strong. Thus, one can approach a critical state in such a system by varying the energy, even though one cannot reach a true metallic state. In Fig. 3 we have an insulating system, the conductance distribution again fitting well to a $\log$-normal distribution. The distribution for the critical state $p_{c}(g)$, shown in Fig. 4 , again has the abrupt cut-off at $\ln (g)=0$. It can be fitted to a skewed $\log$-normal distribution, which is normalized on the interval $(-\infty ; 0]$ rather than all real numbers. Notice that in this case too $p_{c}(g)$ is highly asymmetric and very similar to the case shown in Fig. 11.

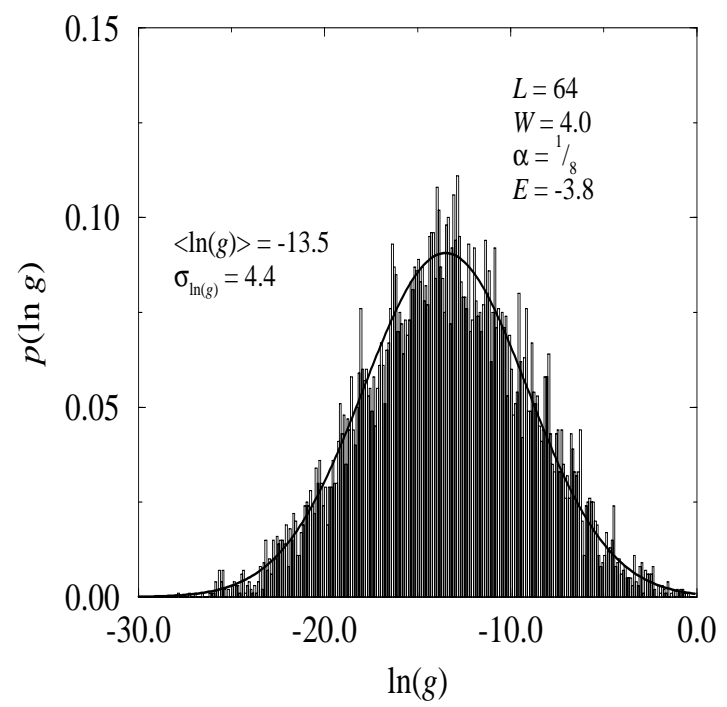

FIG. 3. The conductance distribution of a square system of 64 by 64 lattice sites. For the chosen disorder strength $W=4$ and magnetic flux $\alpha=1 / 8$ states at the energy $E=-3.8$ are well localized. The parameters for the Gaussian fit are given in the graph.

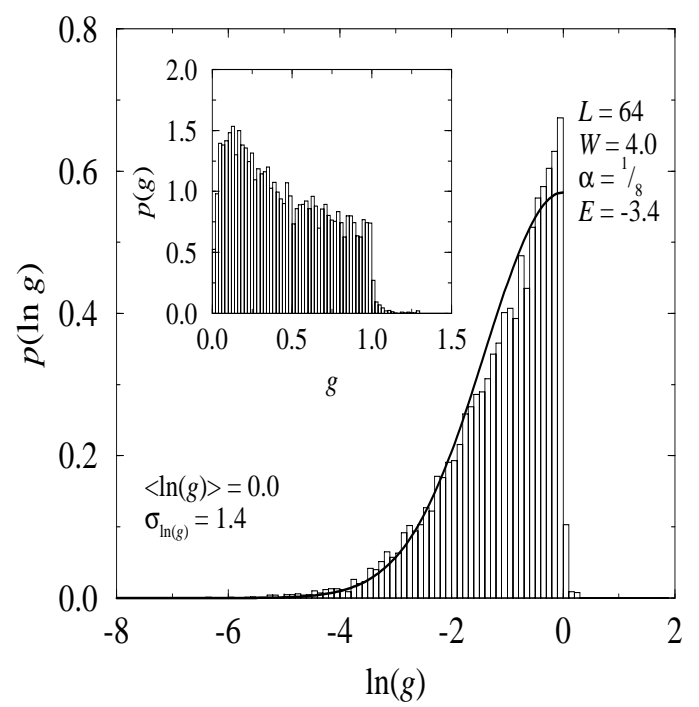

FIG. 4. The conductance distribution of a square system of 64 by 64 lattice sites. For the chosen disorder strength $W=4$ and magnetic flux $\alpha=1 / 8$ states at the energy $E=-3.4$ are critical. The parameters for the fit are given in the graph.

Comparing these results to a $3 \mathrm{~d}$ system without magnetic field, again varying the disorder strength and keeping the energy fixed at $E=0.0$, we find the same qualitative picture: the distribution of the conductance is normal on the metallic side and log-normal on the in- 
sulating side of the transition (see Fig. 5), whereas the critical state is characterized by a cut-off at $\ln (g)=0$ and a skewed $\log$-normal distribution for $\ln (g) \leq 0$ (see Fig. 6).
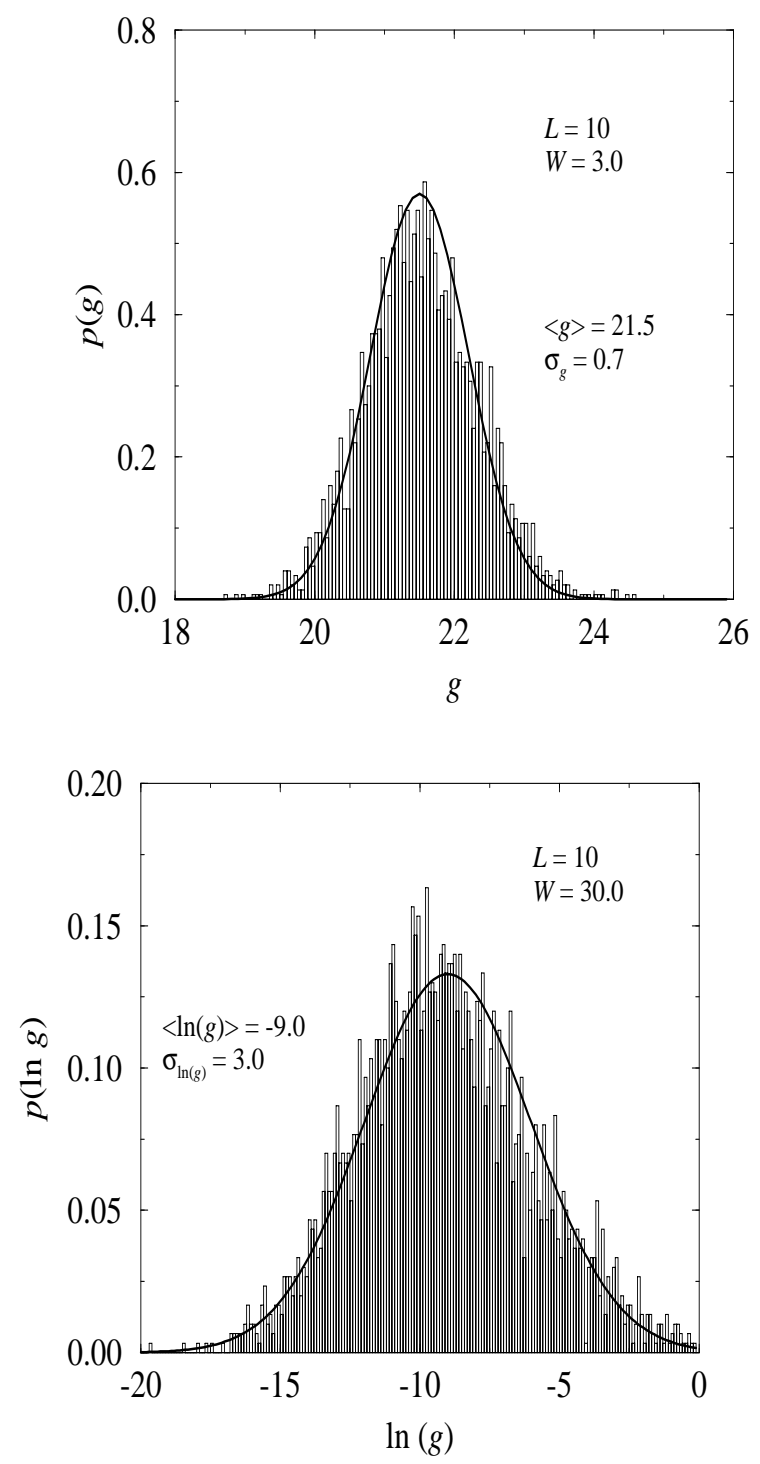

FIG. 5. The conductance distribution of a cubic system of 10 by 10 by 10 lattice sites. Small disorder $(W=3$, left panel) leads to metallic behaviour; strong disorder $(W=30$, right panel) leads to insulating behaviour. The values for the average and standard deviation of the Gaussian fits are given in the graphs.

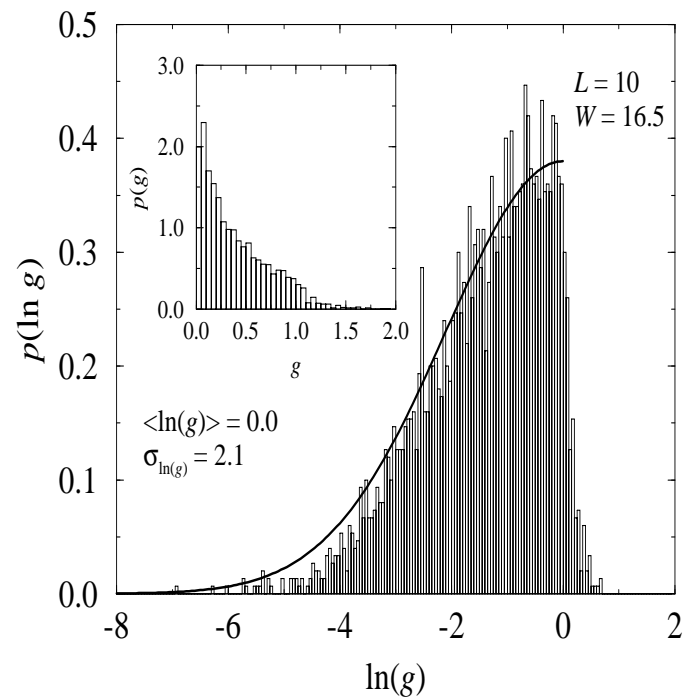

FIG. 6. The conductance distribution of a cubic system of 10 by 10 by 10 lattice sites. The disorder was chosen such that the localization length is close to the system size $L$. The parameters for the fit are given in the graph.

In conclusion, our detailed numerical results show that the probability distribution of the conductance is normal for the extended regime and log-normal for the localized regime. However, at the mobility edge $p(g)$ is highly asymmetric. The form of $p_{c}(g)$ at the critical point is independent of the dimensionality of the system and of the model. This suggests that $p_{c}(g)$ is universal.

Ames Laboratory is operated for the U.S. Department of Energy by Iowa State University under Contract No. W-7405-Eng-82.

${ }^{1}$ C. M. Soukoulis and E. N. Economou, Waves in Random Media 9, 255 (1999) and references therein.

${ }^{2}$ K. Slevin and T. Ohtsuki, Phys. Rev. Lett. 78, 4083 (1997).

${ }^{3}$ C. M. Soukoulis, X. Wang, Q. Li, and M. M. Sigalas, Phys. Rev. Lett. 82, 668 (1999).

${ }^{4}$ P. Markos, Phys. Rev. Lett. 83, 588 (1999).

${ }^{5}$ K. A. Muttalib and P. Wölfle, Phys. Rev. Lett. 83, 3013 (1999).

${ }^{6}$ X. Wang, Q. Li, and C. M. Soukoulis, Phys. Rev. B 58, 3576 (1998).

${ }^{7}$ V. Plerou and Z. Wang, Phys. Rev. B 58, 1967 (1998).

${ }^{8}$ E. N. Economou, Greens Functions in Quantum Physics, $2^{\text {nd }}$ edition (Heidelberg, Springer, 1983).

${ }^{9}$ B. Kramer and A. McKinnon, Rep. Prog. Phys. 56, 1469 (1993) and references therein. 